\title{
Luminescence properties of ZnS phosphor nanocrystals prepared by the laser-induced gas-evaporation method
}

\author{
Masanori Tanaka ${ }^{\text {a) }}$ \\ Single Quantum Dot Project, ERATO, Japan Science and Technology Corporation, 5-9-9 Tokodai, \\ Tsukuba 300-2635, Japan \\ Shinya Sawai \\ Department of Mathematics and Physics, National Defence Academy, Yokosuka 239-8686, Japan \\ Masaya Sengoku \\ Department of Physics, Aichi Medical University, Nagakute-cho, Aichi 480-1103, Japan \\ Manabu Kato \\ Institute of Space and Astronautical Science, Center for Advanced Spacecraft Technology, \\ Sagamihara 229-0022, Japan \\ Yasuaki Masumoto \\ Single Quantum Dot Project, ERATO, Japan Science and Technology Corporation, 5-9-9 Tokodai, \\ Tsukuba 300-2635, Japan and Institute of Physics, University of Tsukuba, Tsukuba 305-8571, Japan
}

(Received 7 February 2000; accepted for publication 16 March 2000)

\begin{abstract}
Nanocrystalline particles of $\mathrm{ZnS}:(\mathrm{Ag}, \mathrm{Al})$ semiconductor phosphor, whose sizes are mostly $3-5 \mathrm{~nm}$ in diameter, are prepared by the gas-evaporation method with $\mathrm{cw} \mathrm{CO}_{2}$ laser heating. The Raman scattering spectrum as well as the transmission electron microscope observation demonstrates that the crystallization of the nanoparticles was caused successfully through the gas-phase condensation. Under irradiation of ultraviolet light, the nanoparticles exhibit blue luminescence, as in the case of the starting material of $\mathrm{ZnS}:(\mathrm{Ag}, \mathrm{Al})$ bulk powder. The peak of the luminescence spectrum of the nanoparticles shifts to lower energy with increasing delay time and also with decreasing excitation intensity, showing that the luminescence originates from the donor-acceptor pair recombination. However, it is concluded that the luminescence of the nanoparticles is not ascribed to the blue $\mathrm{Ag}$ luminescence mechanism responsible for the luminescence of the bulk powder, by taking into account the spatial confinement of an electron trapped at the donor and a hole at the acceptor. It is argued that the luminescence mechanism of the nanoparticles is the so called self-activated luminescence, which involves zinc vacancies. (C) 2000 American Institute of Physics.
\end{abstract}

[S0021-8979(00)06112-0]

\section{INTRODUCTION}

Nanometer-sized crystals show remarkable changes in optical, structural, and electronic properties compared with the bulk crystal, because of the quantum confinement effect and the large surface to volume ratio. ${ }^{1}$ Recently there have been a large number of studies on nondoped semiconductor nanocrystals. ${ }^{1}$ However, optical properties of nanocrystalline semiconductors doped with impurity ions remains largely unexplored despite their potential applications. Accordingly, it is very important to investigate how size reduction of the doped semiconductor affects the optical properties, from the viewpoints of application as well as basic physics. ${ }^{2}$

A representative phenomenon that is often observed in the doped semiconductor is donor-acceptor pair luminescence, which occurs by the electron-hole recombination after trapping of carriers at donors and acceptors. Because $\mathrm{ZnS}$ doped with donors of IIIb and VIIb elements and acceptors of Ib elements exhibits an intense luminescence of this

a) Author to whom correspondence should be addressed; electronic mail: matanaka@sqdp.trc.-net.co.jp type, ${ }^{3,4}$ this is widely used as the phosphors for cathode-ray tube (CRT) displays. These phosphors have particle sizes of a few microns to a few tens of microns. In order to increase the resolution and the brightness of CRT displays under the excitation by the low-energy electron beam, particles of smaller sizes are desirable.

Nanocrystalline particles can be prepared by several methods such as gas evaporation, sputtering, and chemical reaction synthesis. The gas-evaporation method is wellknown to be applicable to many materials irrespective of metals and insulators and or inorganic and organic materials. ${ }^{5,6}$ When a resistance heater is used for the gas evaporation of a compound, there is a possibility of decomposition into the constituents during the heating, because the heating rate is relatively low. In fact, it is known that $\mathrm{ZnS}$ is decomposed into the zinc and sulfur by this method. This difficulty may be circumvented by heating with a high-power laser beam, provided that starting material has an absorption band at the laser wavelength.

In this article, we report the preparation of nanoparticles of $(\mathrm{Ag}, \mathrm{Al})$ doped $\mathrm{ZnS}$ phosphor by the laser-induced gasevaporation method and the optical experiments. From these 
results, the origin of the luminescence of the nanoparticles is discussed.

\section{EXPERIMENT}

The starting material was commercially available (ZnS: $\left(10^{-4} \mathrm{Ag}, 10^{-4} \mathrm{Al}\right)$ bulk phosphor powder. The crystallite size of this powder has distribution ranging from 5 to 20 $\mu \mathrm{m}$. The powder was pressed into a pellet and set on a target holder, which can be moved horizontally, inside an evaporation chamber. The beam of a cw $\mathrm{CO}_{2}$ laser (Nippon Infrared Industries, model NC-1100) with power of about $80 \mathrm{~W}$ was focused on the pellet through a ZnSe lens, and the target materials were evaporated and condensed in an atmosphere of helium gas at a pressure of 1.0 Torr. The particles deposited on the wall of the chamber (or on quartz plates set on the wall of the chamber) were collected and were used for the optical experiments and the observation by transmission electron microscope (TEM).

Luminescence spectra under a cw light excitation were measured using a $27.5 \mathrm{~cm}$ monochromator (Acton Research, Spectrapro-275) equipped with a Si charge-coupled-device (CCD) detector (Princeton Instruments, LN/CCD-1100PF/ UV). The monochromatic light of $330 \mathrm{~nm}$ wavelength, which was obtained by passing the light from a $500 \mathrm{~W}$ xenon lamp through a $25 \mathrm{~cm}$ monochromator (JASCO, CT-25 CPT) and UV band-pass filter (Hoya Glass, U330) was used for the cw excitation. The excitation intensity dependence of the luminescence spectrum was examined under irradiation of the $325.0 \mathrm{~nm}$ line of a $\mathrm{He}-\mathrm{Cd}$ laser (KIMMON Electric, IK3902R-G). Raman scattering spectra were measured by use of a triple-gating polychromator (JASCO, TRS-600/S) with the above CCD detector under the excitation by the 514-5 nm line of a cw $\mathrm{Ar}^{+}$laser (Coherent Innova 90). In this measurement, the spectral resolution was about 0.7 $\mathrm{cm}^{-1}$. Further, time-resolved luminescence spectra were taken with a combination of a $30 \mathrm{~cm}$ monochromator (Acton Research, Spectrapro-300i) and an intensified CCD detector (Princeton Instruments, 1024MG-E/L) operated with a gate width of $0.1 \mu \mathrm{s}$. In this case, the excitation light was a $\mathrm{XeCl}$ excimer laser (Lambda Physik Compex 110)-pumpedBiBuQ dye laser (Lambda Physik, Scanmate) of $10 \mathrm{~Hz}$ repetition rate, about $20 \mathrm{~ns}$ pulse duration, and $380 \mathrm{~nm}$ wavelength. The measurements at low temperature were done using a closed-cycle He refrigator (Daikin, UV202CL). The wavelength calibration of all the CCD detectors was done by the emission lines from atomic vapor lamps. The intensities of the luminescence spectra and the Raman spectra were corrected for the wavelength dependence of the grating efficiency of the monochromators and the response of the CCD detectors.

For the TEM observation, the prepared particles were dispersed in methanol and drops of this dispersion are put on a copper mesh. The electron microscope (Hitachi, $\mathrm{H}-9000 \mathrm{Nar}$ ) was operated at $300 \mathrm{kV}$.

\section{RESULTS AND DISCUSSION}

We could observe many lattice plane images clearly in the TEM images of the prepared $\mathrm{ZnS}:(\mathrm{Ag}, \mathrm{Al})$ sample. A

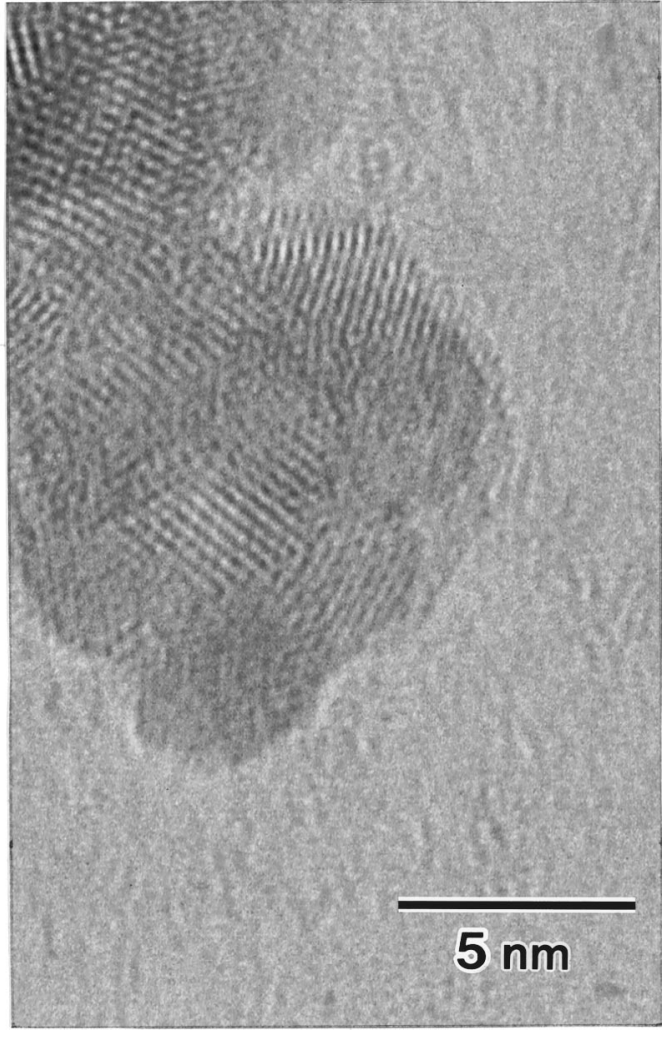

FIG. 1. TEM micrograph of $\mathrm{ZnS}:(\mathrm{Ag}, \mathrm{Al})$ particles grown in helium gas of 1.0 Torr pressure by the laser-induced evaporation method. The magnification is $6 \times 10^{6}$

typical TEM micrograph is shown in Fig. 1. As a result of the measurement of the scales of the lattice plane images, most of the crystallite sizes were found to be in the range of $3-5 \mathrm{~nm}$. Although we also notice in Fig. 1 that the particles may be grains formed from several crystallites touching side to side, the grain sizes (or the degree of the particle aggregation) are believed, from earlier reports, ${ }^{7,8}$ to have increased during the process of TEM sample preparation. Accordingly, the sample treated for the TEM observation was not used for the optical experiments. We could not determine experimentally the impurity densities in the crystallites, because the amounts of the produced powder was too small to analyze them chemically. However, from a calculation based on volume and impurity density in the starting material, many of the crystallites are estimated to contain at most one $\mathrm{Al}$ and one Ag.

In Fig. 2, we show the Raman scattering spectra of the bulk powder and the prepared nanoparticles of $\mathrm{ZnS}:(\mathrm{Ag}, \mathrm{Al})$. Compared with the spectrum of the bulk powder, the peak of the first-order longitudinal optical phonon (1-LO) line of the nanoparticles is shifted by $2.8 \mathrm{~cm}^{-1}$ to lower energy and the linewidth becomes broad. These features can be qualitatively explained by the relaxation of the wave-vector selection rule, which results from the quantum confinement effect of the LO phonon. ${ }^{9,10}$ The Raman spectrum as well as the lattice plane images observed by TEM demonstrate that the decomposition into $\mathrm{Zn}$ and $\mathrm{S}$ was not serious unlike the case of the gas-evaporation method with a resistance heater and the 


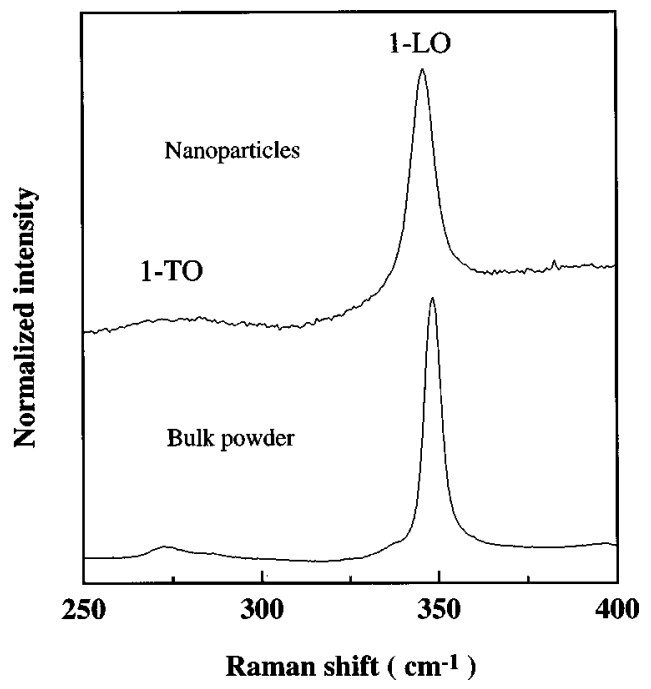

FIG. 2. Raman spectra of the nanoparticles and the bulk powder of $\mathrm{ZnS}:(\mathrm{Ag}, \mathrm{Al})$ at $300 \mathrm{~K} .1-\mathrm{TO}$ and 1-LO denote the first-order transverse and longitudinal optical phonon lines, respectively. The spectral resolution is about $0.7 \mathrm{~cm}^{-1}$. The excitation was done by the $514.5 \mathrm{~nm}$ line of an $\mathrm{Ar}^{+}$ laser.

crystallization of $\mathrm{ZnS}$ was achieved successfully through the gas-phase condensation.

Figure 3 shows the absorption spectrum of the nanoparticles at $300 \mathrm{~K}$. A hump can be seen around $3.8 \mathrm{eV}$ in this figure. In the absorption spectrum of $\mathrm{ZnS}: \mathrm{Cl}$ bulk crystal, a similar hump has been observed at the low-energy side of the interband absorption edge (about $3.7 \mathrm{eV}$ ), ${ }^{12}$ and it has been ascribed to the bound(zinc vacancy)-to-free transition. ${ }^{13}$ Correspondingly, the hump in the absorption spectrum of our nanoparticle sample can be assigned to the free(bound)-tobound(free) transition, while an intense absorption band with an edge at $4.2 \pm 0.1 \mathrm{eV}$, which is located at the high-energy side of the hump, is attributable to the interband transition. Possible candidates for the trap center forming the bound state are silver acceptor and/or zinc vacancy. [In $\mathrm{ZnS}$ :(Ag, $\mathrm{Al}$ ), the silver and the aluminum substitute $\mathrm{Zn}^{2+}$ in the

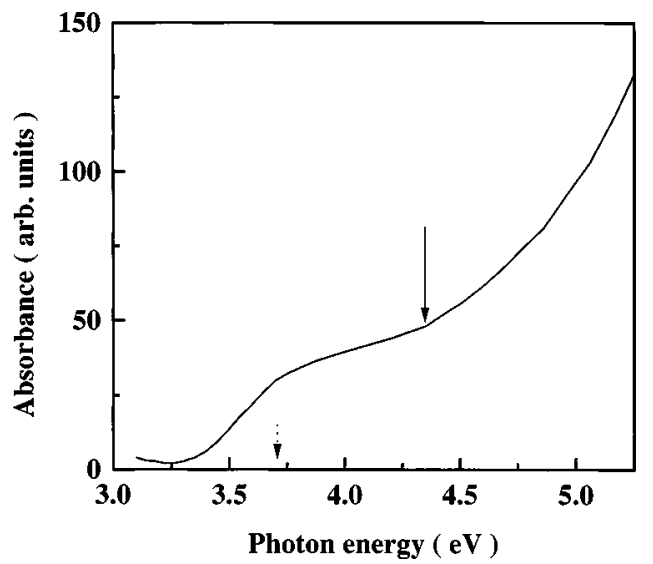

FIG. 3. Absorption spectrum of $\mathrm{ZnS}:(\mathrm{Ag}, \mathrm{Al})$ nanoparticles at room temperature. The solid arrow denotes approximately the band gap energy position in the nanoparticles, from the comparison with the absorption spectrum of bulk $\mathrm{ZnS}: \mathrm{Cl}$ (Ref. 12), while the dotted arrow shows the band gap energy position of bulk $\mathrm{ZnS}$ (Ref. 11).

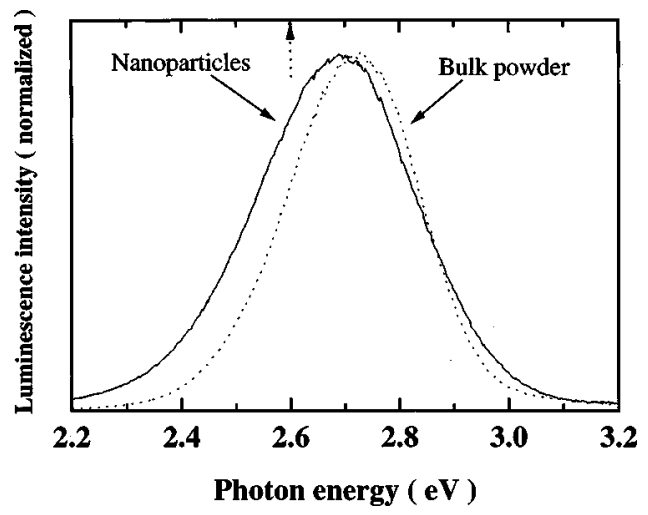

FIG. 4. Luminescence spectra of the nanoparticles and the bulk powder of $\mathrm{ZnS}:(\mathrm{Ag}, \mathrm{Al})$ at $10 \mathrm{~K}$ under the $3.76 \mathrm{eV}$ (wavelength $330 \mathrm{~nm}$ ) excitation. The excitation intensity was the same for both these samples. For comparison, the peak position of the self-activated luminescence in bulk $\mathrm{ZnS}: \mathrm{Al}$ is denoted by the dotted arrow (Refs. 3 and 15).

monovalent and trivalent states, respectively. As a result, $\mathrm{Al}_{\mathrm{Zn}}^{+}$donor and $\mathrm{Ag}_{\mathrm{Zn}}^{-}$acceptor are formed.] The large blueshift of the interband absorption edge relative to that of the bulk crystal shows that the quantum confinement effect for the carriers occurs in the prepared nanoparticle sample., ${ }^{2,8,14}$ Using a variational calculation of Brus, ${ }^{14}$ the average particle size is estimated, from the shift of the absorption band edge, to be about $3 \mathrm{~nm}$ in diameter, which agrees fairly well with the dimensions of the lattice plane images in the TEM images.

Under excitation by ultraviolet light, the nanoparticles of $\mathrm{ZnS}$ :(Ag, Al) exhibit blue luminescence. In Fig. 4, we show the luminescence spectra of the nanoparticles and the bulk powder of $\mathrm{ZnS}:(\mathrm{Ag}, \mathrm{Al})$ at $10 \mathrm{~K}$ under the excitation at 3.76 $\mathrm{eV}$ (wavelength $330 \mathrm{~nm}$ ). These spectra were taken by using the excitation light with the same intensity for both samples. The luminescence spectrum of the bulk powder with a peak at about $2.73 \mathrm{eV}$ (wavelength $454 \mathrm{~nm}$ ) is known as the blue Ag band, ${ }^{3,4}$ which is due to the recombination between an electron trapped at an $\mathrm{Al}_{\mathrm{Zn}}^{+}$donor and a hole at an $\mathrm{Ag}_{\mathrm{Zn}}^{-}$ acceptor. The luminescence spectrum of the nanoparticles is broadened largely to the low-energy side of that of the bulk powder, and its peak is shifted to lower energy by about 0.04 $\mathrm{eV}$ in comparison with that of the bulk powder.

Figure 5 shows the luminescence spectra of $\mathrm{ZnS}$ :( $\mathrm{Ag}$, $\mathrm{Al})$ nanoparticles at various delay times after the pulsed dye laser excitation at $3.26 \mathrm{eV}$ (wavelength $380 \mathrm{~nm}$ ) at $300 \mathrm{~K}$. The peak of the luminescence spectrum is found to be shifted to lower energy with increasing delay time. In addition, the change in the peak energy during decay is larger for the earlier time region, as shown in Fig. 6.

In Fig. 7, we show the decay behaviors at several photon energies within the luminescence band. All the decay curves are nonexponential, and, except for the earliest time region, they obey the relation of power law

$$
I \propto t^{-n} .
$$

Here $I$ and $t$ are the luminescence intensity and the delay time, respectively. As shown in Fig. 8, the value of $n$ increases monotonically with increasing photon energy, corre- 


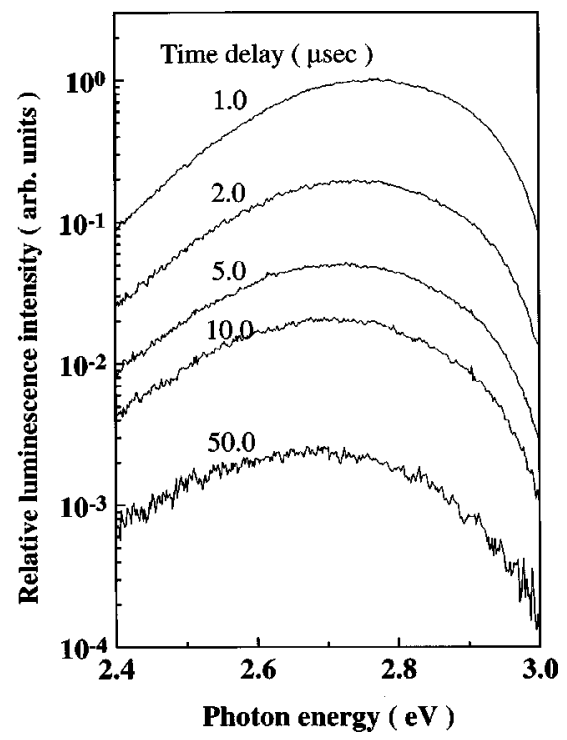

FIG. 5. Luminescence spectra of $\mathrm{ZnS}:(\mathrm{Ag}, \mathrm{Al})$ nanoparticles for various delay times after the pulsed dye-laser excitation at $3.26 \mathrm{eV}$ (wavelength 380 $\mathrm{nm})$. The sample temperature is $300 \mathrm{~K}$.

sponding to the fact that the luminescence peak redshifts as time evolves. Similar luminescence decay behaviors were previously observed for the donor-acceptor pair luminescences in the $\mathrm{ZnS}^{3,4}$ and $\mathrm{GaP}$ bulk crystals. ${ }^{16}$ It was shown theoretically by Thomas et al. ${ }^{16}$ that the decay behavior of the donor-acceptor pair luminescence nearly follows the relation (1) except for the early time region. ${ }^{4}$ Accordingly, the luminescence of the nanoparticle sample can be ascribed to the donor-acceptor pair recombination. Further, the gradual change in the $n$ value with the photon energy in Fig. 8 indicates that a single kind of luminescence mechanism and not plural kinds of mechanisms is responsible for the luminescence band of the nanoparticles.

Figure 9 shows the excitation intensity dependence of the luminescence spectrum of $\mathrm{ZnS}:(\mathrm{Ag}, \mathrm{Al})$ nanoparticles. As the excitation intensity is increased, the luminescence spectral peak is shifted to higher energy. This result is also one of the characteristics of the donor-acceptor pair recombinations expected for the sample where the separation be-

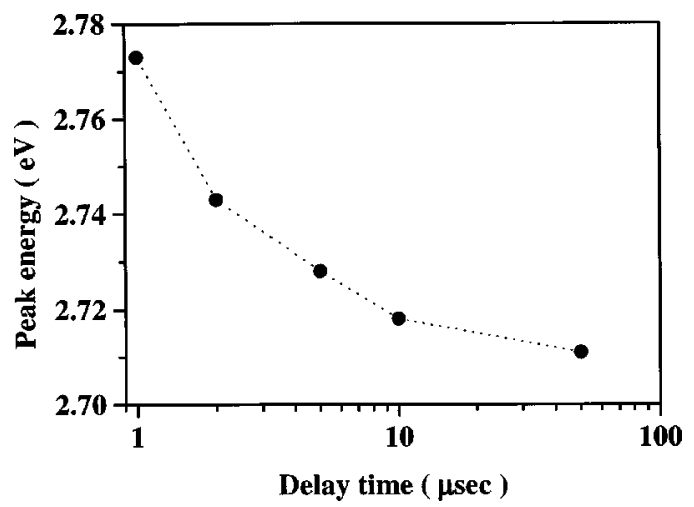

FIG. 6. Peak energies of the time-resolved luminescence spectra of $\mathrm{ZnS}:(\mathrm{Ag}, \mathrm{Al})$ nanoparticles at various delay times. These data were obtained from Fig. 5.

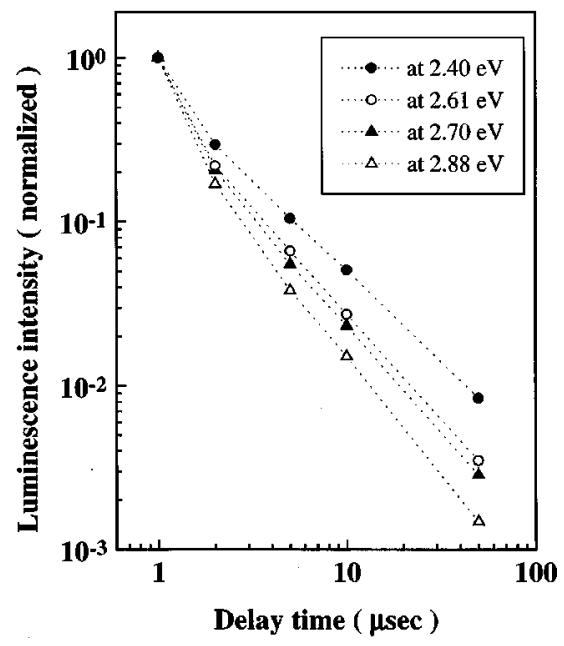

FIG. 7. Decay behaviors at various photon energies in the luminescence band of $\mathrm{ZnS}:(\mathrm{Ag}, \mathrm{Al})$ nanoparticles. These data were obtained from Fig. 5.

tween the donor and the acceptor has a distribution, ${ }^{17}$ and supports the attribution of the observed luminescence to this type of mechanism.

Next, we consider how a reduction in the crystallite sizes affect the donor-acceptor pair luminescence spectrum, in order to examine whether the luminescence of $\mathrm{ZnS}:(\mathrm{Ag}, \mathrm{Al})$ nanoparticles results from the same mechanism as that of the bulk powder, namely, the blue Ag luminescence mechanism. The photon energy emitted by a donor-acceptor pair is expressed by

$$
E(R)=E_{d}-E_{a}+e^{2} /(\epsilon R),
$$

where $E_{a}$ and $E_{d}$ are the energies of the donor and acceptor levels, respectively, $e$ is the electron charge, $\epsilon$ is the static dielectric constant, and $R$ is the separation between the donor and acceptor. The observed donor-acceptor pair luminescence consists of unresolved luminescence lines of various pairs with different $R$ values. Now we assume that the luminescence of the nanoparticles is due to the blue Ag luminescence mechanism. The Ag acceptor level involved in this mechanism is fairly deep (about $0.7 \mathrm{eV}$ above the valence band edge) in bulk $\mathrm{ZnS},{ }^{18}$ and the change in $E_{a}$ is considered

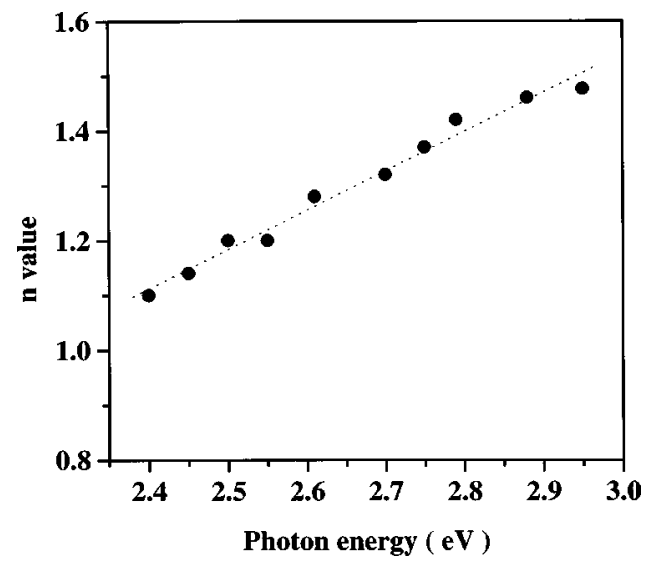

FIG. 8. The $n$ values in the relation (1) at various photon energies in the luminescence band of $\mathrm{ZnS}:(\mathrm{Ag}, \mathrm{Al})$ nanoparticles. These data were obtained from Fig. 5. 


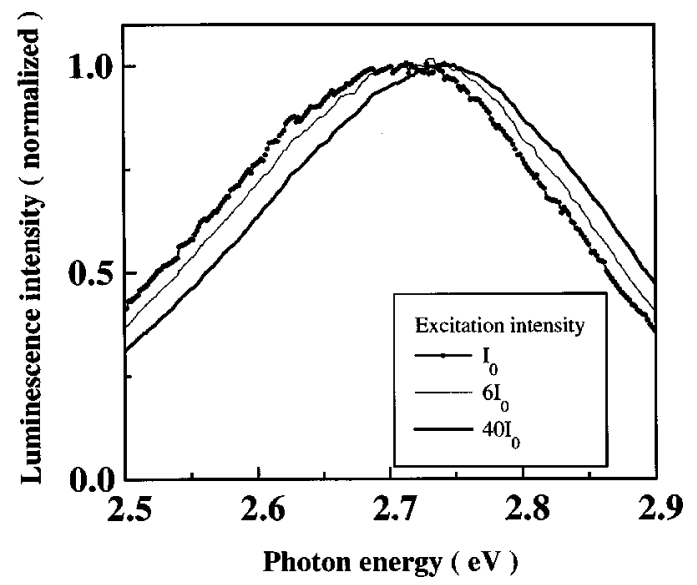

FIG. 9. Luminescence spectra of $\mathrm{ZnS}:(\mathrm{Ag}, \mathrm{Al})$ nanoparticles for various excitation intensities at $10 \mathrm{~K}$. The excitation was done by the $325.0 \mathrm{~nm}$ line (photon energy $3.81 \mathrm{eV}$ ) of a He-Cd laser. $I_{0}$ is the lowest excitation intensity.

to be negligibly small for the size reduction. On the other hand, the aluminum donor in bulk $\mathrm{ZnS}$ has a shallow level $(0.074 \mathrm{eV}$ below the conduction band edge $),{ }^{18}$ and the orbital radius of an electron trapped at the donor is about $1.2 \mathrm{~nm} .{ }^{19}$ Because the value of this radius is fairly close to that of the nanoparticles, $E_{d}$ increases remarkably with decreasing crystallite size, on account of the spatial confinement effect, ${ }^{20-22}$ as in the case of the band gap energy. ${ }^{14}$ As a simple example, let us consider the case where the aluminum donor is located in the center of a spherical $\mathrm{ZnS}$ particle with the diameter 4 $\mathrm{nm}$, which is the approximate average size of our nanocrystals determined from the dimensions of the lattice plane images in the TEM micrograph. In this case, the donor level is estimated to be shifted to higher energy by about $0.1 \mathrm{eV}$, compared with that of the bulk crystal, by a theoretical calculation using the effective mass approximation. ${ }^{21,22}$ Thus, it is predicted that the particle size reduction causes a shift of the donor-acceptor pair luminescence spectrum to the highenergy side. This prediction has been supported experimentally for the donor-acceptor pair luminescence of the $\mathrm{CdS}$ nanocrystals prepared by the chemical method. ${ }^{23}$

Contrary to the above prediction, the luminescence spectrum of our nanoparticles is shifted largely to the low-energy side compared with that of the bulk powder, as seen in Fig. 4. Accordingly, it is not correct to ascribe the blue luminescence of the nanoparticles to the blue Ag luminescence mechanism responsible for the luminescence of the bulk powder. When the density of the IIIb (e.g., Al) or VIIb element donor is fairly low compared with the density of the Ib element acceptor (e.g., Ag) in the bulk $\mathrm{ZnS}$ phosphors, the self-activated blue luminescence, which has a peak at about $2.60 \mathrm{eV}$ (dotted arrow in Fig. 4), is observed predominantly. ${ }^{3,24-26}$ This luminescence is known to result from the recombination of an electron trapped at $\mathrm{Al}_{\mathrm{Zn}}^{+}$with a hole at an acceptor of a nearest-neighbor associate of a zinc vacancy and $\mathrm{Al}_{\mathrm{Zn}}^{+} \cdot{ }^{3,4,24-27}$ Currently, the probable mechanism of the luminescence observed in the nanoparticles is considered to be the self-activated luminescence for the following three reasons: First, this luminescence has the same character of the donor-acceptor pair transition as the self-activated luminescence. Second, the peak position of the blue luminescence of the nanoparticles is higher in energy by about 0.1 $\mathrm{eV}$ than that of the self-activated luminescence observed in the bulk $\mathrm{ZnS}$ powder, which satisfies the requirement of the spatial confinement. Third, it is very probable that zinc vacancies involved in the self-activated luminescence are created in the process of vaporization by laser heating.

The following two of our experimental results will be advantageous for practical applications to CRT displays with a high resolution under the low-energy electron beam excitation. One is that the nanocrystals of $\mathrm{ZnS}$ were obtained easily by the laser-induced gas-evaporation method with no serious decompositions and considerably good crystallinity, as is obvious from the Raman spectrum and the TEM micrograph. The other is that the nanocrystals of $\mathrm{ZnS}:(\mathrm{Ag}, \mathrm{Al})$ show blue luminescence, as in the case of the bulk starting material, which is now put to various practical uses as the blue light emitting phosphors.

\section{SUMMARY}

In summary, we have prepared nanocrystalline particles of $\mathrm{ZnS}$ : $(\mathrm{Ag}, \mathrm{Al})$ phosphor, whose sizes are mostly 3-5 nm in diameter, by the $\mathrm{CO}_{2}$ laser-induced gas-evaporation method. Due to the quantum confinement effect, the band gap energies of the nanoparticles are shifted remarkably to higher energy, compared with that of the bulk $\mathrm{ZnS}$ crystal. The Raman scattering data and the TEM observation showed that the crystallization of the nanoparticles is caused successfully through the gas-phase condensation. Under irradiation of ultraviolet light, the nanoparticles exhibit blue luminescence. From the observation of the shift in luminescence spectrum during decay and with excitation intensity, the blue luminescence has been ascribed to the donor-acceptor pair recombination. The luminescence decay curve has been found to obey the relation of power law at various photon energies within the luminescence band. The mechanism of the luminescence has been concluded to be different from that of the blue Ag luminescence responsible for the starting material of $\mathrm{ZnS}$ :(Ag, Al) bulk powder, by taking into account the spatial confinement effect for the donor level and the acceptor level. Our current hypothesis is that the luminescence of the nanoparticles has the mechanism of the self-activated luminescence.

\section{ACKNOWLEDGMENTS}

One of the authors (M.T.) is indebted to Dr. K. Era of Helios Optical Science Laboratory and Professor Y. Nakanishi of Shizuoka University for informative discussion about ZnS bulk phoshors.

\footnotetext{
${ }^{1}$ H. Gleiter, Prog. Mater. Sci. 33, 223 (1989).

${ }^{2}$ For a review, see J. Lumin. 70 (1996), Special Issue on Spectroscopy of Isolated and Assembled Semiconductor Nanocrystals, edited by L. E. Brus, Al. L. Efros, and T. Itoh.

${ }^{3}$ K. Era, S. Shionoya, and Y. Washizawa, J. Phys. Chem. Solids 29, 1827 (1968).

${ }^{4}$ K. Era, S. Shionoya, Y. Washizawa, and H. Ohmatsu, J. Phys. Chem. Solids 29, 1843 (1968).

${ }^{5}$ R. Uyeda, Prog. Mater. Sci. 35, 1 (1991).

${ }^{6}$ M. Kato, Jpn. J. Appl. Phys. 15, 757 (1976).

${ }^{7}$ W. G. Becker and A. J. Bard, J. Phys. Chem. 87, 4888 (1983).
} 
${ }^{8}$ K. Sooklal, B. S. Cullum, S. M. Angel, and C. J. Murphy, J. Phys. Chem. 100, 4551 (1996).

${ }^{9}$ H. Richter, Z. P. Wang, and L. Ley, Solid State Commun. 39, 625 (1981).

${ }^{10}$ M. Kobayashi, H. Iwata, H. Hanzawa, T. Yoshiue, and S. Endo, Phys. Status Solidi B 198, 515 (1996).

${ }^{11}$ M. M. Firsova, Sov. Phys. Solid State 16, 35 (1974)

${ }^{12} \mathrm{~S}$. Shionoya, in Luminescence of Inorganic Solids, edited by P. Goldberg (Academic, New York, 1966), Chap. 4, p. 205.

${ }^{13}$ S. Oda and H. Kukimoto, J. Lumin. 18\&19, 829 (1979).

${ }^{14}$ L. E. Brus, J. Chem. Phys. 80, 4403 (1984).

${ }^{15} \mathrm{~K}$. Era (private communication). The peak position of the self-activated luminescence of bulk $\mathrm{ZnS}: \mathrm{Al}$ varies with the excitation intensity, because this luminescence is due to the donor-acceptor pair recombination. However, it is certain that, under the excitation of the $\mathrm{ZnS}: \mathrm{Al}$ and $\mathrm{ZnS}:(\mathrm{Ag}, \mathrm{Al})$ bulk crystals with the same intensity, the peak of the self-activated luminescence in the former sample is lower in energy by about $0.13 \mathrm{eV}$ than that of the blue Ag luminescence in the latter sample.

${ }^{16}$ D. G. Thomas, J. J. Hopfield, and W. M. Augustyniak, Phys. Rev. 140, A202 (1965).
${ }^{17}$ P. Y. Yu and M. Cardona, Fundamentals of Semiconductors (Springer, Berlin, 1996), Chap. 7, p. 333.

${ }^{18}$ Physics of II-VI and I-VII Compounds, Semimagnetic Semiconductors, edited by O. Madelung, M. Schulz, and H. Weiss, Landolt-Börnstein, New Series, Group III, Vol. 17, Pt. B (Springer, Berlin, 1982).

${ }^{19}$ H. Kukimoto, S. Shionoya, T. Koda, and R. Hioki, J. Phys. Chem. Solids 29, 935 (1968).

${ }^{20}$ D. S. Chuu, C. M. Hsiao, and W. N. Mei, Phys. Rev. B 46, 3898 (1990).

${ }^{21}$ N. Matsuura, M. Tanaka, S. Nair, and Y. Masumoto, 53rd Annual Meeting, 1998 Meeting Abstract of the Physical Society of Japan 53, 199 (1998).

${ }^{22}$ M. Tanaka, N. Matsuura, and Y. Masumoto (unpublished).

${ }^{23}$ N. Chestnoy, T. D. Harris, R. Hull, and L. E. Brus, J. Phys. Chem. 90, 3393 (1986).

${ }^{24}$ J. S. Prener and D. J. Weil, J. Electrochem. Soc. 106, 409 (1959).

${ }^{25}$ W. van Gool, Phillips Res. Rep. Suppl. 3, 1 (1961).

${ }^{26}$ K. Urabe and S. Shionoya, J. Phys. Soc. Jpn. 24, 543 (1968).

${ }^{27}$ J. S. Prener and F. E. Williams, J. Chem. Phys. 25, 361 (1956). 